\title{
Structural characterization of polysaccharide from jujube (Ziziphus jujuba Mill.) fruit
}

\author{
Xiaolong $\mathrm{Ji}^{1}$, Yanqiu Cheng ${ }^{1}$, Jingyuan Tian ${ }^{1}$, Siqi Zhang ${ }^{1}$, Yongshuai Jing $^{2^{*}}$ and Miaomiao Shi ${ }^{1 *}$
}

\begin{abstract}
Background: Jujube (Ziziphus jujuba Mill.) fruit is one of the largest productions in China and its increasing production has drawn considerable attention from researchers. Polysaccharide is one of the most abundant components of jujube, and it represents a major group of biolotegically active constituents. This study intended to investigate the special structure of a homogeneous acidic polysaccharide (PZMP4) produced from Ziziphus Jujuba cv. Muzao fruit using novel methods, including DEAE-Sepharose Fast Flow and Sephacryl S-300 column chromatography.

Results: The structure of PZMP4 was determined via high-performance gel permeation chromatography (HPGPC), gas chromatography (GC), Fourier transform infrared spectroscopy (FT-IR), methylation analysis, nuclear magnetic resonance spectroscopy (NMR), scanning electron microscopy (SEM), and atomic force microscopy (AFM). The results reveal that PZMP4 with a molecular weight of $27.90 \mathrm{kDa}$ was composed of rhamnose, arabinose, mannose, glucose, galactose, and galacturonic acid at a ratio of 2.32:2.21:0.22:0.88:2.08:8.83. Advanced structural analysis revealed a netted structure with molecular aggregates of PZMP4. Structural features demonstrated that the basic backbone of PZMP4 appeared to mainly consist of $(1 \rightarrow 4)$-linked GalpA with three branches bonded to 0-3 of $(1 \rightarrow 3)$-linked Araf, $(1 \rightarrow 2)$-linked Rhap, and terminated with GalpA.
\end{abstract}

Conclusions: PZMP4's unique structure could imply distinct bioactivities and have considerable utilization in functional food.

\section{Highlights}

Structural characteristics of PZMP4 were analyzed by HPGPC, GC, FT-IR, GC-MS, NMR, SEM and AFM. PZMP4 mainly consisted of $(1 \rightarrow 4)$-linked GalpA with three branches boned to O-3.

The branches included ( $1 \rightarrow 3)$-linked Araf, $(1 \rightarrow 2)$-linked Rhap, and terminated with Gal $p$ A.

Keywords: Jujube, Polysaccharide, Purification, Structural characterization

*Correspondence: cjys1985@126.com; chengzi3090@163.com

${ }^{1}$ College of Food and Bioengineering, Zhengzhou University of Light Industry, Zhengzhou 450001, People's Republic of China

${ }^{2}$ College of Chemistry and Pharmaceutical Engineering, Hebei University of Science and Technology, Shijiazhuang 050018, People's Republic of China

\section{Background}

Jujube, (Ziziphus jujuba Mill.), a member of the family Rhamnaceae, is widely used as a medicinal herb. It is predominant in subtropical Asian and European countries, particularly in China $[1,2]$. The $Z$. jujuba fruit is nutrient-dense, with carbohydrates accounting for the majority (55-85\%). It also has moisture (25-30\%), crude protein (2.9-6.6\%), crude fiber (2.4-8.4\%), crude fat (0.4-1.0\%), and several other vitamins and minerals [3]. 
Furthermore, numerous essential functional components present in jujube, including polysaccharides, flavonoids, saponins, and cyclic nucleotides, have been demonstrated to account for a variety of biological activities, such as anti-inflammatory, anti-cancer, and anti-oxidant activities, as well as hematopoietic function, neurobeneficial properties, and intestinal flora regulation [4].

Of the various functional components in $Z$. jujuba fruit, polysaccharide is especially important because of its bioactivities and large cellular concentrations. It is mainly composed of different ratios of monosaccharides and glycosidic bonds [5]. The activities of polysaccharides of $Z$. jujuba are determined by their molecular weights and chemical structures. An increased galacturonic acid concentration could result in enhancing antioxidant activity [6]. The structural-physicochemical properties and bioactivities of polysaccharides vary greatly among different $Z$. jujuba varieties [1]. Our research efforts have contributed to a better understanding of the structural basis of jujube polysaccharides [7-9].

In this present study, an acidic polysaccharide (PZMP4) was isolated from Ziziphus Jujuba cv. Muzao and characterized by HPGPC, GC, AFM, SEM, NMR spectroscopy, FT-IR spectroscopy, and methylation analysis.

\section{Materials and methods Materials}

The Z. Jujuba cv. Muzao fruits were supplied by the Jia County of Shaanxi. Sephacryl S-300 and DEAE-Sepharose Fast Flow cellulose were provided by GE Healthcare Life Sciences. Standard monosaccharides were obtained from Sigma Chemical Co. All additional chemicals utilized in the experiments were of analytical grade.

\section{Polysaccharide isolation}

The production of crude polysaccharide from $Z$. Jujuba $c v$. Muzao (ZMP) from jujube fruit at the red and ripened stage was performed as previously described [10, 11]. After re-dissolution, the ZMP was added to a DEAESepharose FF column equalized with $0.4 \mathrm{M} \mathrm{NaCl}$ and a Sephacryl S-300 column balanced with distilled water. It was then gathered, concentrated, and lyophilized for the production of designative PZMP4 purified polysaccharides [7].

\section{General methods}

The carbohydrate content was determined by the phenol-sulfuric acid method with glucose as the standard [12]. The Bradford method with bovine serum albumin as the reference was used to assess the protein content [13]. The Folin-Ciocalteu colorimetric method was used to quantify the value of the total phenol content [14].
To identify and quantify PZMP4 monosaccharide, GC analysis was performed as reported previously [15]. HPGPC on an Agilent-LC 1200 instrument equipped with a TSK-gel G3000PWxl $(7.8 \mathrm{~mm} \times 300 \mathrm{~mm})$ column was used to analyze the homogeneity and average molecular weight of PZMP4 $[7,16]$.

The IR spectra of PZMP4 were obtained by the $\mathrm{KBr}$ disc method, with $400-4000 \mathrm{~cm}^{-1}$ range for the FT-IR spectrometer. The one- and two-dimensional NMR spectra of PZMP4 were acquired with a Bruker AVIII-600 NMR spectrometer [8].

We first performed carboxyl reduction with 1-ethyl3-(3-dimethylaminopropyl) carbodiimide and $\mathrm{NaBD}_{4}$, and then further performed hydrolysis with trifluoroacetic acid, reduction with $\mathrm{NaBH}_{4}$, and acetylation with acetic anhydride. The resultant partially methylated alditol acetate derivatives were identified by their retention times and electron ionization spectra on a GCMSQP2010A instrument equipped with a DB-17MS capillary column $(60.0 \mathrm{~m} \times 0.25 \mathrm{~mm} \times 0.25 \mu \mathrm{m})$ [8].

The surface morphology of PZMP4 was examined using an S-4800 SEM (Japan) under $10 \mathrm{kV}$ accelerating voltage. PZMP4 was dissolved in distilled water, dropped on the surface of a mica carrier, and then dried at $70{ }^{\circ} \mathrm{C}$ under ambient pressure [8]. The AFM images were taken with an Agilent 5500 atomic force microscope (USA) in tapping mode [7].

\section{Results and discussion}

\section{Preliminary PZMP4 characterization}

ZMP was isolated from Z. Jujuba cv. Muzao fruit and extracted by ultrasonic-assisted extraction, ethanol precipitation, deproteination, dialysis, and lyophilization. Further purification was subsequently done using a DEAE Sepharose Fast Flow column $(2.6 \mathrm{~cm} \times 100 \mathrm{~cm})$, which was eluted with phosphate-buffered saline (20 mM, pH 6.0), and $0.4 \mathrm{M} \mathrm{NaCl}$ solution at a flow rate of $1.5 \mathrm{~mL} / \mathrm{min}$. We determined the elution by observing phenol-sulfuric acid, and then passed it through a Sephacryl S-300 column $(2.6 \mathrm{~cm} \times 100 \mathrm{~cm})$ with deionized water for further purification. As a result, a single elution peak named PZMP4 was obtained. The total carbohydrate content of PZMP4 was found to be $92.64 \%$. The protein and total phenol contents of PZMP4 were $3.09 \%$, and $0.95 \%$, respectively, higher than the acidic (PZMP2-2) polysaccharide from Z. Jujuba cv. Muzao [8].

As shown in Fig. 1A, HPGPC revealed that the acidic polysaccharide PZMP4 was homogeneous, with only one symmetrical absorption peak. A standard curve of the logarithm of relative molecular weight associated with elution time $(t)$ was created using a serious detraining, shown as the following: $\lg \mathrm{Mw}=-0.3259 t+10.9495\left(R^{2}=99.57 \%\right)$. On the 


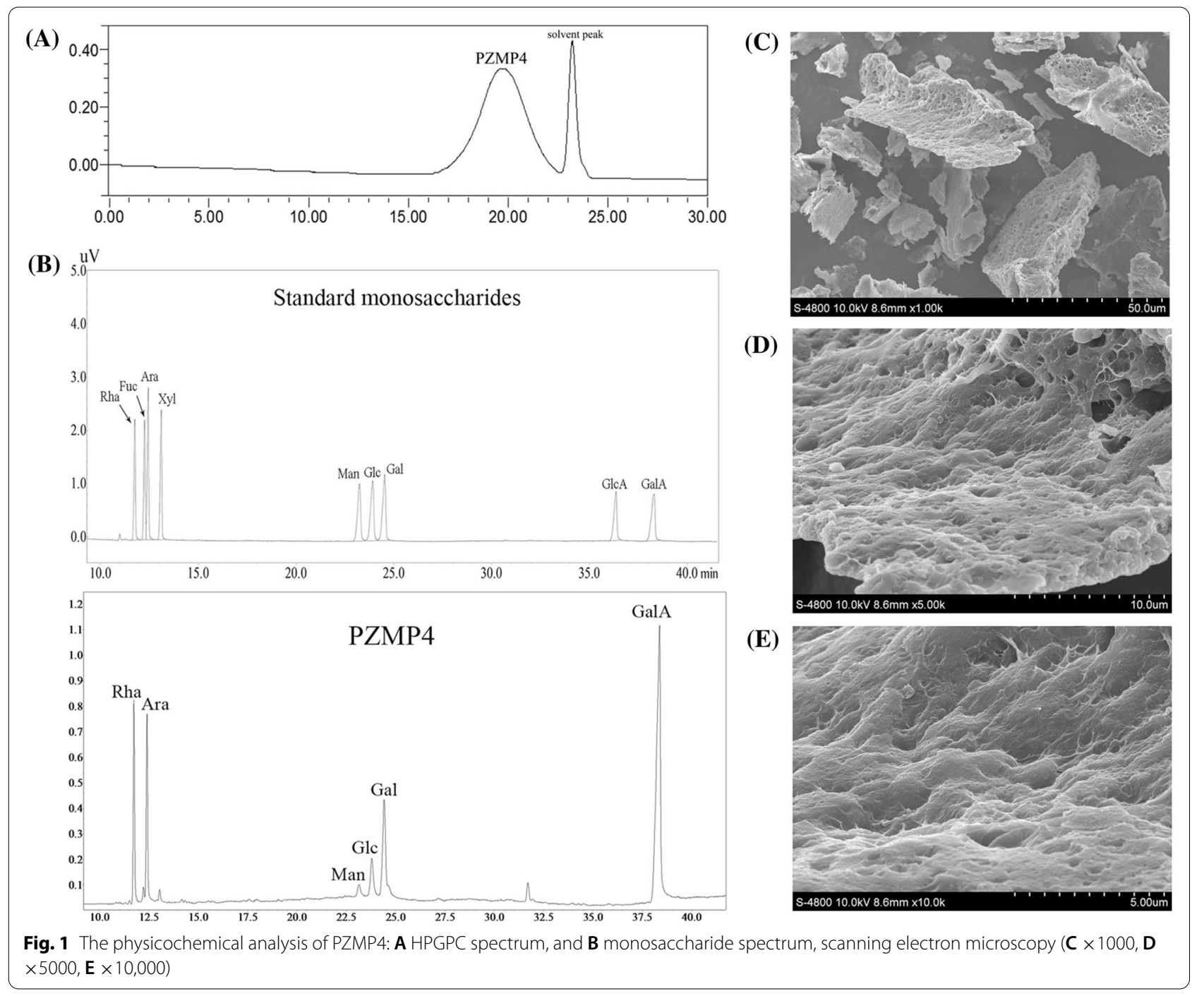

basis of the equation, the average molecular weight of PZMP4 was estimated to be $27.90 \mathrm{kDa}$ with a retention time of $19.983 \mathrm{~min}$. This acidic jujube polysaccharide fraction had a molecular weight similar to HJP-4 ( $Z$. Jujuba cv. Hamidazao polysaccharide) [1].

GC was used to determine the monosaccharide component of PZMP4. The hydrolysate of PZMP4 consisted of six monosaccharides, including rhamnose, arabinose, mannose, glucose, galactose, and galacturonic acid (Fig. 1B). Their ratios were 2.32:2.21:0.22:0.88:2.08:8.83, suggesting that PZMP4 was an acidic heteropolysaccharide. Furthermore, the results indicated that rhamnose, arabinose, and galacturonic acid accounted for the majority of the total polysaccharide content. However, the result was different for Z. Jujuba cv. Hamidazao polysaccharide fractions; this may be caused by different raw materials, as well as extraction and purification methods $[1,5]$.

\section{FT-IR spectrum analysis}

The functional groups and chemical bonds of PZMP4 were further analyzed by FT-IR (Additional file 1: Fig. $\mathrm{S} 1 \mathrm{~A})$. The $\mathrm{O}-\mathrm{H}$-stretching vibration and the $\mathrm{C}-\mathrm{H}$ stretching vibration were represented by distinct bands in the 3411 and $2937 \mathrm{~cm}^{-1}$ regions, respectively. The absorbances at 1741 and $1244 \mathrm{~cm}^{-1}$ demonstrated the existence of uronic acid, which corroborated the results of the uronic acid assay $[8,17]$. The symmetrical $\mathrm{C}=\mathrm{O}$-stretching vibrations were verified by the high peak at $1610 \mathrm{~cm}^{-1}$ [18]. The $1415 \mathrm{~cm}^{-1}$ peak represented the characteristic absorption of $\mathrm{C}-\mathrm{H}$ bands, while the $1099 \mathrm{~cm}^{-1}$ peak indicated the pyranose form's 
$\mathrm{C}-\mathrm{O}$-stretching vibrations [8]. The slight characteristic absorptions at $800-900 \mathrm{~cm}^{-1}$ may indicate the existence of $\alpha$ - and $\beta$-configurations [19]. Consequently, FT-IR analysis of PZMP4 revealed absorption peaks of typical plant polysaccharides.

\section{Methylation analysis}

PZMP4 was methylated and reacted with trifluoroacetic acid. The resultant partly methylated alditol acetates were examined by gas chromatography-mass spectrometry (GC-MS). Table 1 shows the linkage types and molar ratio percentages of PZMP4 according to the Complex Carbohydrate Research Center (CCRC) Spectral Database for PMAA. PZMP4 contained four main glycosidic linkages, 1,2-linked Rhap, 1-linked Gal $p \mathrm{~A}$, 1,3-linked Araf, and 1,3,4-linked GalpA with molar percentage ratios of 1.64:1.00:1.52:4.50, thus, implying that the PZMP4's backbone could only be of 1,4-linked GalpA residues, with branches connected to the $O-3$ position for some residues. The structure of PZMP4 was further supported by NMR spectroscopy.

\section{NMR analysis}

To determine the detailed structure of PZMP4, onedimensional and two-dimensional NMR spectra were used for further study. The $\mathrm{C} / \mathrm{H}$ chemical shifts of several glycosidic bonds were consistent with the previous literature; the data are shown in Fig. 2 and Table 2. The ${ }^{1} \mathrm{H}$-NMR spectrum of PZMP4 (Fig. 2A) displays four main anomeric proton signals at $\delta 4.87,5.00,4.82$, and 5.00/5.05, which were designated as $\mathrm{A}, \mathrm{B}, \mathrm{C}$, and D, respectively. $\mathrm{H}-2, \mathrm{H}-3, \mathrm{H}-4$, and $\mathrm{H}-5$ of 1,3,4-linked GalpA residues were responsible for the significant peaks in the 3.74-5.06 ppm range. The ${ }^{13} \mathrm{C}$ NMR spectrum (Fig. 2B) revealed six anomeric signals resonating at $101.89,70.79 / 70.93,79.42,84.05,73.41 / 73.04$, and $173.68 / 173.80 \mathrm{ppm}$. According to previous results in the literature, the relevant anomeric carbon signals of tagged residues in the ${ }^{1} \mathrm{H}$ and ${ }^{13} \mathrm{C}$ NMR spectra were attributed to data in the 2D NMR spectra [20,21]. From the chemical shift data in the COSY (Additional file 2: Fig. S2B), NOESY (Additional file 2: Fig. S2C), ${ }^{1} \mathrm{H} /{ }^{1} \mathrm{H}$ TOCSY (Additional file 2: Fig. S2D), HSQC (Additional file 2: Fig.
S2E), and HMBC spectra (Additional file 2: Fig. S2F), the proton and carbon assignments of four main residues in PZMP4 are presented in Table 2.

The signal at $\delta_{\mathrm{H}} 5.00$ was determined from the chemical shift of the anomeric proton of residue $B$. The equivalent signal in the anomeric carbon was $\delta_{\mathrm{C}} 102.36$. The $\delta_{\mathrm{C}}$ $71.45 / \delta_{\mathrm{H}} 3.91, \delta_{\mathrm{C}} 70.79 / \delta_{\mathrm{H}} 3.63(3.66), \delta_{\mathrm{C}} 71.59 / \delta_{\mathrm{H}} 3.96$, $\delta_{\mathrm{C}} 74.21 / \delta_{\mathrm{H}} 4.32$, and $\delta_{\mathrm{C}} 178.21$ signals were allocated to $\mathrm{C}-2, \mathrm{C}-3, \mathrm{C}-4, \mathrm{C}-$, and $\mathrm{C}-6$ of residue $\mathrm{B}$, respectively $[22,23]$. The anomeric proton of residue $C$ had a chemical shift of $4.82 \mathrm{ppm}$, whereas the anomeric carbon had a chemical shift of $102.36 \mathrm{ppm}$. The COSY and TOCSY spectra were used to identify the other protons in residue C. According to HSQC, the other comparable carbon and hydrogen signals were 84.19 (4.11/4.04), 71.45 (3.86), 74.85 (3.66), 71.60 (3.69), and 19.44 (1.15) ppm. According to the NMR data, the chemical shifts of this residue were identical to that of $\alpha-1,2$-linked Rhap [24, $25]$. HSQC indicated the other carbon and hydrogen signals with 110.24 (5.00/5.05), 81.61 (4.20), 86.77 (3.94), 83.74 (4.02), and 63.96 (3.62) ppm. From the NMR data, the chemical shifts of this residue were identical to that of $\alpha$-1,3-linked Gal $p[26,27]$.

HMBC, COSY, and NOESY could determine the glycosidic linkages between sugar residues. Hence, with these techniques, the intra-residue connections were determined and are listed in Table 2. As the HMBC spectrum shows, some inter-residual cross-peaks were identified: A C-3 to D H-1, A C-4 to D H-1, A C-4 to B H-1, D C-3 to D H-1, A C-4 to D H-3, A C-3 to C $\mathrm{H}-2$, and A C-1 to $\mathrm{D} \mathrm{H}-1$. In addition, in the COSY spectrum, certain interresidual cross-peaks were also recognized: $\mathrm{C} / \mathrm{D} \mathrm{H}-1$ to C/D H-2, A/B H-2 to A/B H-3, A/B/C/D H-3 to A/B/C/D $\mathrm{H}-4$, and $\mathrm{C} / \mathrm{D} \mathrm{H}-4$ to $\mathrm{C} / \mathrm{D} \mathrm{H}-5$. A/C H-1 to A H-3 were detected in the NOESY spectrum [28].

According to the monosaccharide composition of PZMP4, combined with the results of FT-IR, GCMS, 1D and 2D NMR analyses, it was determined that PZMP4 was mainly composed of $\rightarrow 4)$-Gal $p \mathrm{~A}-(1 \rightarrow$ backbone, with a branching point at the $O-3$ position consisting of Araf, Rhap, and GalpA residues.

Table 1 Methylation analysis data forPZMP4

\begin{tabular}{|c|c|c|c|c|c|}
\hline Peak no. & Residues & $\begin{array}{l}\text { Retention time } \\
\text { (min) }\end{array}$ & Methylated sugars & Linkage patterns & $\begin{array}{l}\text { Relative } \\
\text { amount } \\
\text { (mol\%) }\end{array}$ \\
\hline 1 & $C$ & 38.941 & 3,4-Me2-Rhap & $\rightarrow 2)-\mathrm{a}-\mathrm{L}-\mathrm{Rhap}-(1 \rightarrow$ & 18.95 \\
\hline 2 & B & 39.572 & 2,3,4,6-Me4-GalpA & a-D-GalpA-(1 $\rightarrow$ & 11.54 \\
\hline 3 & $\mathrm{D}$ & 40.142 & 2,4,5-Me3-Araf & $\rightarrow 3)$-a-L-Araf-(1 $\rightarrow$ & 17.56 \\
\hline 4 & A & 66.252 & 2,6-Me2-GalpA & $\rightarrow 3,4)-a-D-G a l p A-(1 \rightarrow$ & 51.95 \\
\hline
\end{tabular}






Fig. 2 The NMR spectra of PZMP4 in $\mathrm{D}_{2} \mathrm{O} . \mathbf{A}^{1} \mathrm{H}$ spectra; $\mathbf{B}^{13} \mathrm{C}$ spectra

Table 2 Assignments of $1 \mathrm{H}$ and 13C NMR spectra for PZMP4

\begin{tabular}{|c|c|c|c|c|c|c|c|c|}
\hline Residues & Linkage & & 1 & 2 & 3 & 4 & 5 & 6 \\
\hline \multirow[t]{2}{*}{ A } & \multirow[t]{2}{*}{$\rightarrow 3,4)-\mathrm{a}-\mathrm{D}-\mathrm{Gal} p \mathrm{pA}-(1 \rightarrow$} & C & 101.89 & $70.79 / 70.93$ & 79.42 & 84.05 & $73.41 / 73.04$ & $173.68 / 173.80$ \\
\hline & & $\mathrm{H}$ & 4.87 & 3.74 & 3.87 & 4.04 & $5.06 / 5.00$ & \\
\hline \multirow[t]{2}{*}{ B } & \multirow[t]{2}{*}{ a-D-GalpA- $(1 \rightarrow$} & $C$ & 102.36 & 71.45 & 70.79 & 71.59 & 74.21 & 178.21 \\
\hline & & $H$ & 5.00 & 3.91 & $3.63 / 3.66$ & 3.96 & 4.32 & \\
\hline \multirow[t]{2}{*}{ C } & \multirow[t]{2}{*}{$\rightarrow$ 2)-a-L-Rhap-(1 $\rightarrow$} & C & 102.36 & 84.19 & 71.45 & 74.85 & 71.60 & 19.44 \\
\hline & & $\mathrm{H}$ & 4.82 & $4.11 / 4.04$ & 3.86 & 3.66 & 3.69 & 1.15 \\
\hline \multirow[t]{2}{*}{ D } & \multirow[t]{2}{*}{$\rightarrow 3)$-a-L-Araf-(1 $\rightarrow$} & C & 110.24 & 81.61 & 86.77 & 83.74 & 63.96 & \\
\hline & & $\mathrm{H}$ & $5.00 / 5.05$ & 4.20 & 3.94 & 4.02 & 3.62 & \\
\hline
\end{tabular}

\section{Morphological properties}

Different morphological properties are key components that contribute to the complexity of polysaccharide forms. SEM, as a microscopic-molecular-morphology observation technique, is frequently used to characterize the surface morphology of polysaccharides [29]. 
The SEM images of PZMP4 demonstrated the disparate morphological characteristics (Fig. 1C-E). PZMP4 had a rough surface and a netted structure due to molecular aggregation, which was most likely induced by a variety of hydroxyl and carboxyl groups [30].

Another important tool is AFM, which could provide both 2D images and 3D surface images of plant polysaccharides. Additional file 1: Figure S1B, C depicted the AFM findings of PZMP4. We observed an irregular special block structure for PMP4 indicating the state of aggregation, which was compatible with the SEM images. Higher hydroxyl and carbonyl group contents of plant polysaccharide chains appeared to strengthen the intermolecular and intramolecular interactions, and thereby resulted in the strong molecular chain aggregation [31]. The molecules of PZMP4 had a tendency to form a stacked and aggregated structure to be stable.

\section{Conclusions}

A new acidic polysaccharide (PZMP4) from Ziziphus Jujuba cv. Muzao fruit was successfully purified and characterized. PZMP4 was a homogeneous polysaccharide with a molecular weight of $27.90 \mathrm{kDa}$. It was mainly composed of rhamnose, arabinose, mannose, glucose, galactose, and galacturonic acid at a molar ratio of 2.32:2.21:0.22:0.88:2.08:8.83. Methylation and NMR spectroscopy suggested that the main backbone of PZMP4 was composed of $(1 \rightarrow 4)$-linked GalpA, with three branches attached to $O-3$ of $(1 \rightarrow 3)$-linked Araf, $(1 \rightarrow 2)$-linked Rhap, and terminated with GalpA. Notably, the unique structure of PZMP4 indicated that it could possess valuable bioactivities and have wide applications in food industries.

\begin{abstract}
Abbreviations
HPGPC: High-performance gel permeation chromatography; GC: Gas chromatography; FT-IR: Fourier transform infrared spectroscopy; NMR: Nuclear magnetic resonance spectroscopy; SEM: Scanning electron microscopy; AFM: Atomic force microscopy; ZMP: Crude polysaccharide from Zizyphus Jujuba cv. Muzao; TA: Trifluoroacetic acid; GC-MS: Gas chromatography-mass spectrometry.
\end{abstract}

\section{Supplementary Information}

The online version contains supplementary material available at https://doi. org/10.1186/s40538-021-00255-2.

Additional file 1: Figure S1. The physicochemical analysis of PZMP4: (A) FI-IR spectrum, (B) Atomic force microscope planar image, (C) Threedimensional image of atomic force microscope.

Additional file 2: Figure S2. The NMR spectra of PZMP4 in $D_{2} O$. (A) DEPT135 spectra, (B) COSY spectra, (C) NOESY spectra, (D) ${ }^{1} \mathrm{H} / 1 \mathrm{H}$ TOCSY spectra, (E) HSQC spectra, (F) HMBC spectra.

\section{Acknowledgements}

We are grateful to the platform of College of Food and Bioengineering, Zhengzhou University of Light Industry.

\section{Authors' contributions}

Conceptualization and methodology: JX; software and validation: $\mathrm{CY}$; investigation, resources, and data curation: TJ; writing original draft preparation: ZS; writing review and editing: JY; visualization and supervision: JX and SM. All authors have agreed to the published version of the manuscript. All authors read and approved the final manuscript.

\section{Funding}

This work was funded by the Natural Science Foundation of Henan Province (212300410297), Basic Research Plan of Higher Education School Key Scientific Research Project of Henan Province (21A550014), Doctoral Research Foundation of Zhengzhou University of Light Industry (2020BSJJ015), Fourth Chinese Materia Medica Resources Survey (Z135080000022), and Key Research and Development Program of Hebei Province (20370509D).

\section{Availability of data and materials}

The datasets used and/or analyzed during the current study are available from the corresponding author on reasonable request.

\section{Declarations}

Ethics approval and consent to participate

Not applicable.

Consent for publication

Not applicable.

\section{Competing interests}

The authors declare that they have no competing interests.

Received: 13 July 2021 Accepted: 7 September 2021

Published online: 06 October 2021

\section{References}

1. Yang Y, Qiu Z, Li L, Vidyarthi SK, Zheng Z, Zhang R. Structural characterization and antioxidant activities of one neutral polysaccharide and three acid polysaccharides from Ziziphus jujuba cv. Hamidazao: a comparison. Carbohydr Polym. 2021;261:117879.

2. Ji XL, Hou CY, Gao YG, Xue YQ, Yan YZ, Guo XD. Metagenomic analysis of gut microbiota modulatory effects of jujube (Ziziphus jujuba Mill.) polysaccharides in a colorectal cancer mouse model. Food Funct. 2020;11:163-73.

3. Gao QH, Wu CS, Wang M. The jujube (Ziziphus jujuba Mill.) fruit: a review of current knowledge of fruit composition and health benefits. J Agric Food Chem. 2013;61:3351-63.

4. Chen J, Tsim KWK. A review of edible jujube, the Ziziphus jujuba fruit: a heath food supplement for anemia prevalence. Front Pharmacol. 2020;11:593655.

5. Ji X, Peng Q, Yuan Y, Shen J, Xie X, Wang M. Isolation, structures and bioactivities of the polysaccharides from jujube fruit (Ziziphus jujuba Mill.): a review. Food Chem. 2017;227:349-57.

6. Chang SC, Hsu BY, Chen BH. Structural characterization of polysaccharides from Zizyphus jujuba and evaluation of antioxidant activity. Int J Biol Macromol. 2010;47:445-53.

7. Ji X, Liu F, Peng Q, Wang M. Purification, structural characterization, and hypolipidemic effects of a neutral polysaccharide from Ziziphus Jujuba Cv. Muzao. Food Chem. 2018;245:1124-30.

8. Ji X, Zhang F, Zhang R, Liu F, Peng Q, Wang M. An acidic polysaccharide from Ziziphus Jujuba cv. Muzao: purification and structural characterization. Food Chem. 2019;274:494-9.

9. Ji X, Yan Y, Hou C, Shi M, Liu Y. Structural characterization of a galacturonic acid-rich polysaccharide from Ziziphus Jujuba cv. Muzao. Int J Biol Macromol. 2020;147:844-52. 
10. Ji X, Peng Q, Li H, Liu F, Wang M. Chemical characterization and antiinflammatory activity of polysaccharides from Zizyphus jujube cv. Muzao. Int J Food Eng. 2017. https://doi.org/10.1515/ijfe-2016-0382.

11. Qin Z, Liu HM, Cheng XC, Wang XD. Effect of drying pretreatment methods on structure and properties of pectins extracted from Chinese quince fruit. Int J Biol Macromol. 2019;137:801-8.

12. Dubois M, Gilles KA, Harmilton JK, Rebers PA, Smith F. Colorimetric method for determination of sugars and related substances. Anal Chem. 1956:28:350-6.

13. Bradford MM. A rapid and sensitive method for the quantitation of microgram quantities of protein utilizing the principle of protein binding. Anal Biochem. 1976;72:248-54

14. Li S, Shah NP. Characterization, antioxidative and bifidogenic effects of polysaccharides from Pleurotus eryngii after heat treatments. Food Chem. 2016;197:240-9.

15. Wang X, Zhang L, Wu J, Xu W, Wang X, Lu X. Improvement of simultaneous determination of neutral monosaccharides and uronic acids by gas chromatography. Food Chem. 2017;220:198-207.

16. Liu XX, Liu HM, Yan YY, Fan LY, Qin GY. Structural characterization and antioxidant activity of polysaccharides extracted from jujube using subcritical water. LWT Food Sci Technol. 2019;117:108645.

17. Chen Y, Xie MY, Nie SP, Li C, Wang YX. Purification, composition analysis and antioxidant activity of a polysaccharide from the fruiting bodies of Ganoderma atrum. Food Chem. 2008:107:231-41.

18. Vasilieva T, Sigarev A, Kosyakov D, Ul'yanovskii N, Anikeenko E, Chuhchin D. Formation of low molecular weight oligomers from chitin and chitosan stimulated by plasma-assisted processes. Carbohydr Polym. 2017;163:54-61

19. Ren $G, X u L, L u T$, Yin J. Structural characterization and antiviral activity of lentinan from Lentinus edodes mycelia against infectious hematopoietic necrosis virus. Int J Biol Macromol. 2018;115:1202-10.

20. Zhang XH, Liu LN, Lin CW. Structural features, antioxidant and immunological activity of a new polysaccharide (SP1) from sisal residue. Int J Biol Macromol. 2013;59:184-91.

21. Zhan R, Xia L, Shao JH, Wang C, Chen DF. Polysaccharide isolated from Chinese jujube fruit (Zizyphus jujuba cv. Junzao) exerts anti-inflammatory effects through MAPK signaling. J Funct Foods. 2018;40:461-70.
22. Hu HB, Liang HP, Wu Y. Isolation, purification and structural characterization of polysaccharide from Acanthopanax brachypus. Carbohydr Polym. 2015:127:94-100.

23. Zhao ZH, Dai H, Wu XM, Chang HT, Gao XM, Liu MJ. Characterization of a pectic polysaccharide from the fruit of Ziziphus jujuba. Chem Nat Compd. 2007:43(4):374-6

24. Li N, Liu X, He XX, Wang SY, Cao SJ, Xia Z. Structure and anticoagulant property of a sulfated polysaccharide isolated from the green seaweed Monostroma angicava. Carbohydr Polym. 2017:159:195-206.

25. Ji XL, Hou CY, Shi MK, Yan YZ, Liu YQ. An insight into the research concerning Panax ginseng CA Meyer polysaccharides: a review. Food Rev Int. 2020. https://doi.org/10.1080/87559129.2020.1771363.

26. Cardoso SM, Silva AMS, Coimbra MA. Structural characterisation of the olive pomace pectic polysaccharide arabinan side chains. Carbohydr Res. 2002;337:917-24.

27. Ji XL, Peng BX, Ding HH, Cui BB, Nie H, Yan YZ. Purification, structure and biological activity of pumpkin (Cucurbita moschata) polysaccharides: a review. Food Rev Int. 2021. https://doi.org/10.1080/87559129.2021.19049 73

28. Hou CY, Yin MS, Lan P, Wang HR, Nie H, Ji XL. Recent progress in the research of Angelica sinensis (Oliv.) Diels polysaccharides: extraction, purification, structure and bioactivities. Chem Biol Technol Agric. 2021;8:13.

29. Xu Y, Liu G, Yu Z, Song X, Li X, Yang Y. Purification, characterization and antiglycation activity of a novel polysaccharide from black currant. Food Chem. 2016;199:694-701.

30. Ji X, Hou C, Yan Y, Shi M, Liu Y. Comparison of structural characterization and antioxidant activity of polysaccharides from jujube (Ziziphus jujuba Mill.) fruit. Int J Biol Macromol. 2020;149:1008-18.

31. Liu W, Wang H, Yu J, Liu Y, Lu W, Chai Y. Structure, chain conformation, and immunomodulatory activity of the polysaccharide purified from Bacillus Calmette Guerin formulation. Carbohydr Polym. 2016;150:149-58.

\section{Publisher's Note}

Springer Nature remains neutral with regard to jurisdictional claims in published maps and institutional affiliations.

\section{Submit your manuscript to a SpringerOpen ${ }^{\circ}$ journal and benefit from:}

- Convenient online submission

- Rigorous peer review

- Open access: articles freely available online

- High visibility within the field

- Retaining the copyright to your article

Submit your next manuscript at springeropen.com 